\title{
Modeling of Flexible Beam Using System Identification Approach
}

\author{
Noor Fadhilah Mat Ros, Mohd Sazli Saad, Mohd Zakimi Zakaria, Intan Zaurah Mat Darus
}

\begin{abstract}
This paper presents the modeling of the flexible beam using system identification method. Due to great attention of flexible structures in most applications, vibration becomes the main problem regarding the dynamic of the structures. In controlling vibration, the system necessarily needs a good model. Therefore, the aim of the work described in this paper is to obtain a dynamic model of the flexible beam. By considering a discrete time form for the system, an autoregressive with exogenous input (ARX) model structures was selected. A square wave signal has been used as an input signal to excite the beam in order to collect input-output data from the experiment. Recursive least square (RLS) estimation algorithm is used to estimate the model parameters based on different model orders. The model validation was done by comparing the measured output against the predicted output in terms of the closeness of both outputs via mean square error (MSE) value. The final result shows the model with the 12 th order has the lowest error.
\end{abstract}

Keywords - Flexible beam, Modeling, System identification.

\section{INTRODUCTION}

Nowadays the needs of flexible structures are increased due to demand of the lightweight system. In addition, the area of research also grew extensively on how to establish more reliable, lightweight and efficient flexible structures. To date, the material of the structures used in the industries is much lighter and thin. In a matter of fact, even it is extremely popular in industries, but it may lead to unwanted vibration. Indeed, the active vibration control methods are required to adapt the dynamic response of the system. Thus, it is vital to develop a good model in order to control vibration effectively [1]. The problem of finding a good model for the dynamic of systems often occurred in engineering applications. One way to solve this problem is through system identification [2].

System identification is one of the famous methods in modeling of the system. It is a process of obtaining a mathematical model of a system by using input-output data from the experimental study [3]. The general procedure of system identification is represented in Fig. 1 [4].

Noor Fadhilah Mat Ros, Universiti Malaysia Perlis, Malaysia, email id: noor_fadhilah@ymail.com

Dr. Mohd Sazli Saad., Universiti Malaysia Perlis, Malaysia, sazlisaad@unimap.edu.my

Mohd Zakimi Zakaria, Universiti Malaysia Perlis, Malaysia

Intan Zaurah Mat Darus, Universiti Teknologi Malaysia

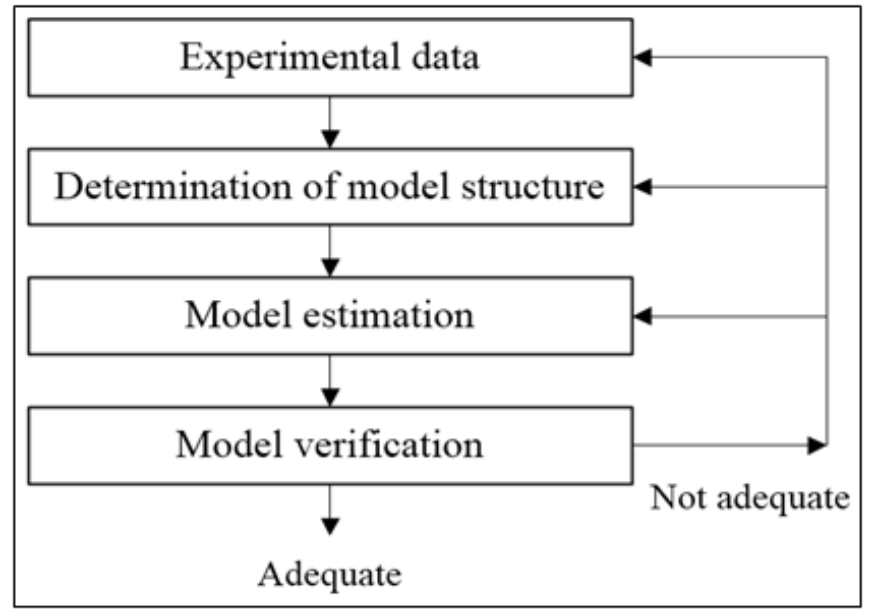

Fig.1. System identification procedure [4]

At the first stage, the related data regarding input and output is extracted from a designed experimental rig. Next, the model structure and its order to represent the system are chosen. Later, the model of the system is estimated by using parameter estimation techniques. Afterward, the validation of the model of the system will be conducted to check whether the model is good enough or not. If the validation is failed, then the whole process should be repeated by obtaining the new set of data [1].

System identification is a very robust method of finding the model of the system. Even though the approach has been used by many researchers in many engineering application, a lot of attention are still being received nowadays [5]. Besides, this approach is suitable for both simple and complex systems [3]. In modeling of flexible beam and plate structures, system identification is often applied. Several studies developed the model of the structure and then the model used to design the controller [6],[7]. The result shows that the good model contributes to the efficiency of vibration control.

Certain researchers developed the model of the structures to compare with the different method of identification. Darus and Tokhi [8] compared parametric and non-parametric identification of flexible plate and applying several estimation methods. Tavakolpour [9] and Hadi et al. [5] developed a model of rectangular plate by comparing conventional and modern parameter estimation methods. The models from each method are compared in terms of their performance. Afshari et al. [3] only focused on modeling of flexible beam by using parametric identification and then simplifying the complex model to simple model. Zakaria et al. [10] modeled flexible beam which is treated as non-linear system and considering multi-objective function for estimation. Other than that, system identification also found to be used in certain applications such 
as flexible single link system [11], automotive engine fuelled [12], internal combustion engine [13], rotor bearing foundation system [14], control of gas bearings [15] and turbojet engine [16]. From the review, system identification is obviously seen to be used successfully in modeling of any system and becomes one of the preferred methods.

In this study, the system identifications of a flexible beam were conducted using recursive least square (RLS) in parameter estimation. The goal is to obtain the good model with minimum error. The experimental rig is set up to obtain the data set through excitation signal. Then, the autoregressive with exogenous inputs (ARX) is selected as a model structure. The parameters of the model estimated using RLS and the performance of the model is evaluated in terms of mean square error value in model validation for different model orders.

\section{II.EXPERIMENTAL SETUP}

An experimental rig of a flexible beam is developed in Fig. 2 is for the resonance test and input-output data collection. The rig of smart beam consists of an aluminum beam bonded with collocated piezoelectric patches which act as disturbance actuator and sensor. The beam is considered as a cantilever which is one end of beam is clamped by the holder. Piezoelectric disturbance actuator generated the unwanted vibration to the structure. The piezoelectric sensor used for sensing the deflection of beam when it has been applied a disturbance and signals are transmitted to computer control system via data acquisition system (DAQ).

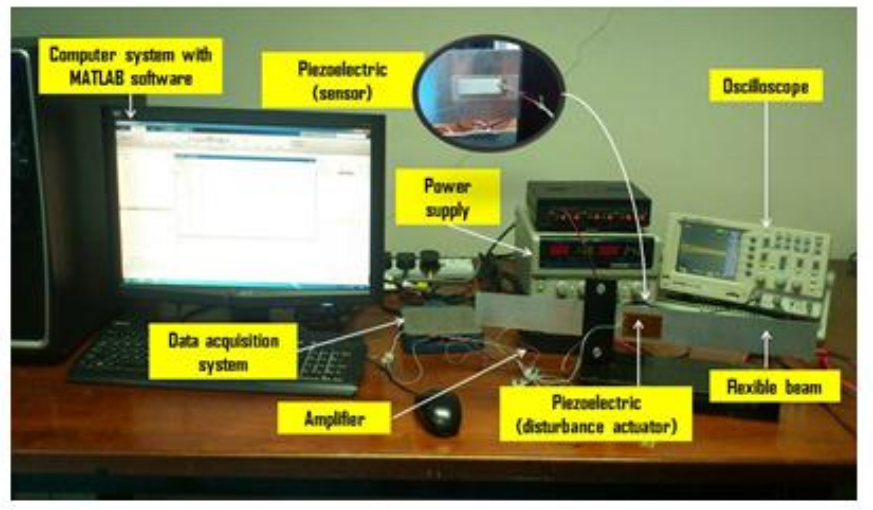

Fig.2. Experimental rig of flexible beam system TABLE I

PROPERTIES OF ALUMINUM FLEXIBLE BEAM

\begin{tabular}{|c|c|c|}
\hline & & \\
PARAMETER & VALUE & UNIT \\
\hline Length & 300 & $\mathrm{~mm}$ \\
\hline Width & 50 & $\mathrm{~mm}$ \\
\hline Thickness & 0.5 & $\mathrm{~mm}$ \\
\hline Young modulus & 70 & $\mathrm{Gpa}$ \\
\hline Density & 2700 & $\mathrm{Kg} / \mathrm{m}^{3}$ \\
\hline
\end{tabular}

\section{SYSTEM IDENTIFICATION}

System identification is modeling of a flexible beam using input-output data from the experiment. Generally, the step involved in system identification is experimental data collection, model structure selection, parameter estimation and model validation. The model is developed based on auto- regressive with exogenous inputs (ARX) structure and model parameters are estimated by a recursive least square algorithm.

\section{A. Model Structure}

The model structure represented the mathematical model of the system to be modeled. The beam is modeled based on second order transfer function. Equations (1) and (2) below are the transfer function of flexible beam bonded with piezoelectric actuator and sensor in continuous time and discrete time for second order system respectively [6]:

$$
\begin{gathered}
\frac{y(t)}{u(t)} \sum_{i=1}^{N} \frac{b_{1 i} s+b_{2 i}}{s^{2}+2 \xi_{i} \omega_{i} s+\omega_{i}^{2}} \\
\frac{y(t)}{u(t)}=\sum_{i=1}^{N} \frac{b_{1 i} z^{-1}+b_{2 i} z^{-2}}{1+a_{1 i} z^{-1}+a_{2 i} z^{-2}}
\end{gathered}
$$

where $y(t)$ is the output signal, $u(t)$ is the input signal, $N$ is the number of modes, $i$ is $i$-th mode of vibration, $b_{1}$ and $b_{2}$ are locations of zeros of the transfer function, $\xi$ is a damping ratio for $i$-th mode of vibration and $\omega_{i}$ is natural frequency of vibrating system. The poles in the transfer function represent the mode frequencies. Equation 2 is expanded to form ARX model which written as follows:

$$
\frac{y(t)}{u(t)}=\frac{b_{1} z^{-1}+b_{2} z^{-2}+\cdots b_{n b} z^{-n b}}{1+a_{1} z^{-1}+a_{2} z^{-2} \ldots a_{n a} z^{-n a}}
$$

where $n a$ and $n b$ are the order of the structure, $b_{n b}$ and $a_{n a}$ are the parameters need to be estimated by algorithms to find the value of estimated output. For this study, the model is built by considering a 4th model order up to 12th order for each estimation method.

\section{B. Recursive least squares (RLS)}

The recursive least square algorithm is a method which used an iterative refinement technique to continuously estimate the parameters using existing parameters and information obtained from the continuous operation of the system. RLS estimates the current parameter vector $\hat{\theta}(i)$ based on the previous value of estimated vector $\hat{\theta}(i-1)[2]$. All four equations presented below are the algorithm of RLS.

$$
\begin{gathered}
\hat{\theta}(i)=\hat{\theta}(i-1)+K(i) * E(i) \\
K(i)=\frac{\lambda^{-1} P(i-1) x(i)}{1+\lambda^{-1} x(i)^{T} P(i-1) x(i)} \\
P(i)=\lambda^{-1} P(i-1)-\lambda^{-1} K(i) x(i)^{T} P(i-1) \\
E(i)=y(i)-x(i)^{T} \theta(i-1)
\end{gathered}
$$

where $\hat{\theta}(i)$ is current parameter vector, $K(i)$ is least square weighting factor, $P(i)$ is a matrix that proportional to variance of previously estimated vector, $E(i)$ is current estimation error, $x(i)$ is the regression vector, $\hat{\theta}(i-1)$ is previous estimated 
vector, $\lambda$ is a forgetting factor, $y(i)$ and $x(i)$ is the system output and input respectively. Fig. 3 shows the schematic representation of iterative process of recursive parameter estimation.

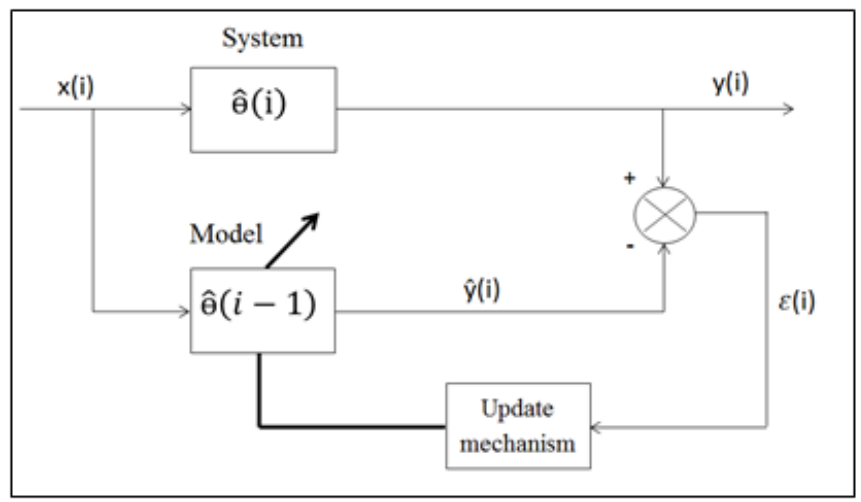

Fig.3. Iterative process of recursive parameter estimation

At each iteration, the new estimate parameter $\hat{\theta}(i)$ is calculated based on the recent values of $x(i)$ and $y(i)$. The system output $y(i)$ is one-time step behind the input $x(i)$. The model based on past information summarized in $\hat{\theta}(i-1)$ is used to obtain an estimate $\hat{y}(i)$ of current output. This is then compared with measured output $y(i)$ to generate error $\varepsilon(i)$. Model parameters are adjusted by an update mechanism derived from the measured quality of model (error). This in turn, generates an update to the model which corrects $\hat{\theta}(i-1)$ to the new value of $\hat{\theta}(\mathrm{i})$.

\section{RESUlTS AND DisCUSSION}

\section{A. Experimental result}

The resonance test and input-output data collection have been established through experimental study. The purpose of resonance test is to find the natural frequencies of vibrated beam. The surface of a beam was applied an impact using rubber hammer as a signal and it is allowed to vibrate freely after the impact. The two dominant modes obtained are first and second modes with frequencies of $4.395 \mathrm{~Hz}$ and $24.66 \mathrm{~Hz}$ respectively. These values have the most significant and highest amplitude compared to other modes.

The square wave signal is used as external excitation to the beam. The values of natural frequencies from resonance test are combined together to generate the square wave signal through Matlab Simulink. The square wave signal which acts as input used to excite the beam and the output data is captured via the displacement of the beam during the vibration (refer Fig. 4).

Once the data set is obtained, the smoothing process is done to smooth the response data by removing the noise and spikes existed. The bandstop filter is applied to reject a band of frequencies, while passing all others. Only the two modes desired as shown in resonance test. The value lies between first and second modes are needed to be rejected. The first 1000 samples of data are used for parameter estimation and another 1000 samples are for validation. Fig. 5 shows the vibration signal from beam excitation within 2000 samples data.

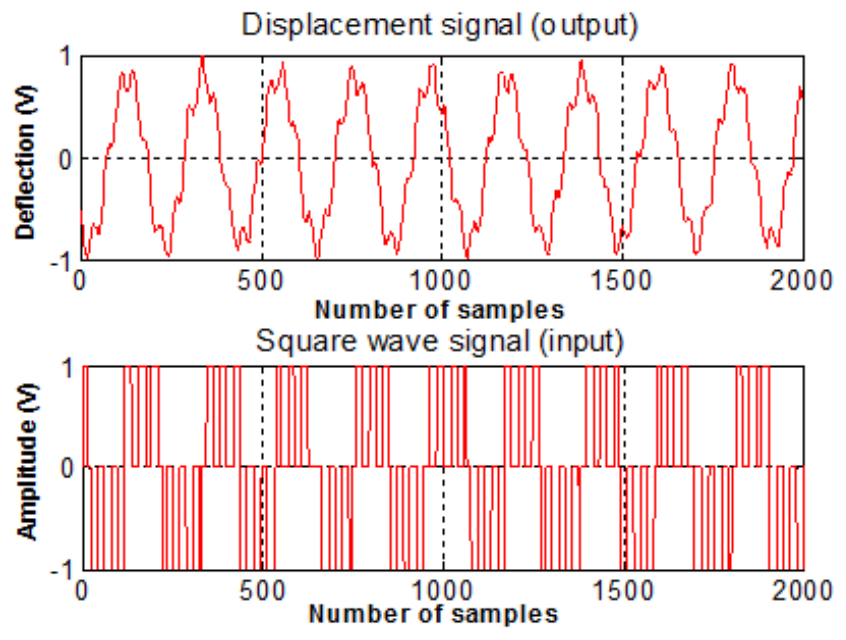

Fig.4. Input and output data from experiment

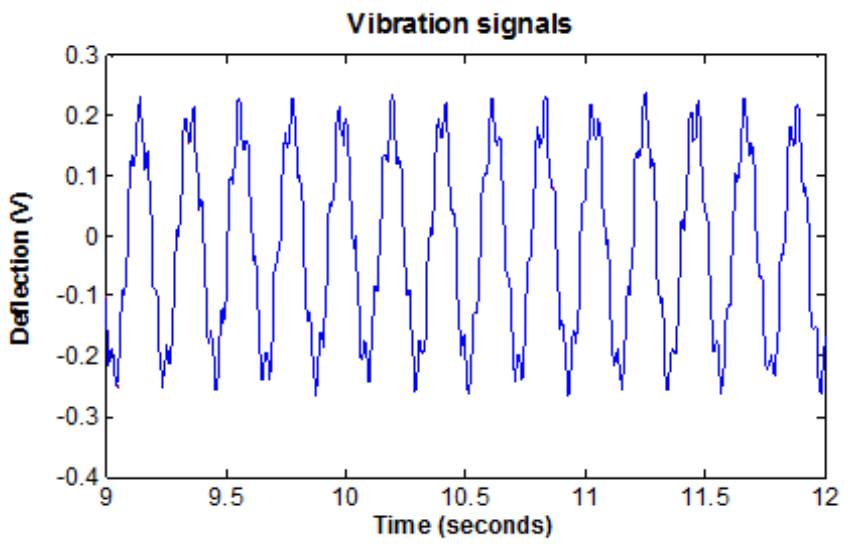

Fig.5. Vibration signal plotted from output data

The displacement signal is plotted in the frequency spectrum to ensure that the beam has the same value of frequencies with the resonance test. From frequency response in Fig. 6, it can be observed that the measured resonance output from the experiment is in agreement with resonance test $(4.395 \mathrm{~Hz}$ and $24.66 \mathrm{~Hz}$ ). The first and second modes are mostly significant with the obviously visible amplitude.

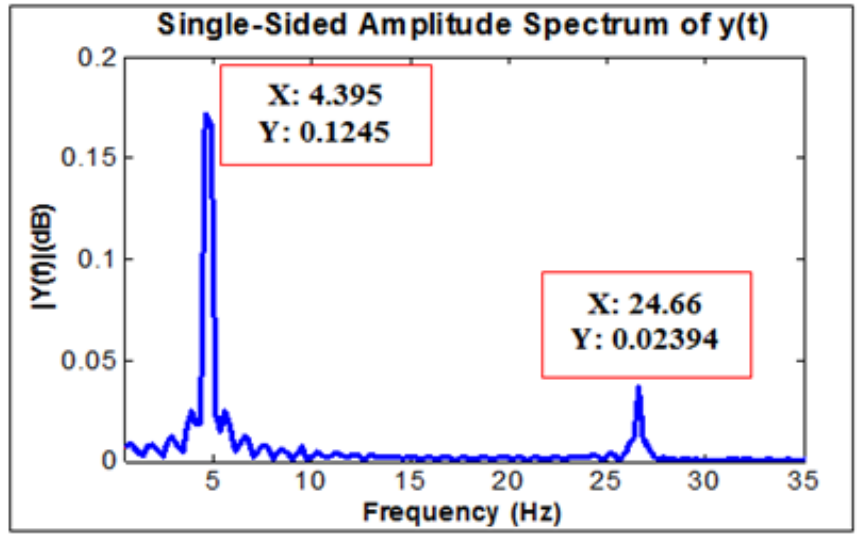

Fig.6. Measured resonance frequency of beam from excitation signal 


\section{B. Parameter estimation and model validation}

Parameter estimation is obtaining the coefficients of the model structure of the flexible beam system. The estimation process usually involves processing input and the corresponding output signals of the plant by estimation algorithm. The estimation of the model is using recursive least square (RLS) method with 1000 iterations and the stopping criterion is the maximum iterations.

The sampling rate used for the estimation is $1 \mathrm{kHz}$. The model is developed by considering 4th order up to 12 th model orders to compare the order with the smallest mean square error (MSE) value. The following Table 2 shows the MSE for each order. The highest model order of 12th showed the lowest MSE. Fig. 7 displays the convergence profile of estimated parameters for 12 th order. It can be seen that the parameters converged rapidly until 1000th iteration

TABLE II

MEAN SQUARE ERROR FOR DIFFERENT ORDERS

\begin{tabular}{|c|c|}
\hline Model Order & Mean Square Error (MSE) \\
\hline 12 & $6.78 \times 10^{-5}$ \\
\hline 10 & $7.11 \times 10^{-8}$ \\
\hline 8 & $9.82 \times 10^{-5}$ \\
\hline 6 & $1.86 \times 10^{-4}$ \\
\hline 4 & $2.00 \times 10^{-4}$ \\
\hline
\end{tabular}

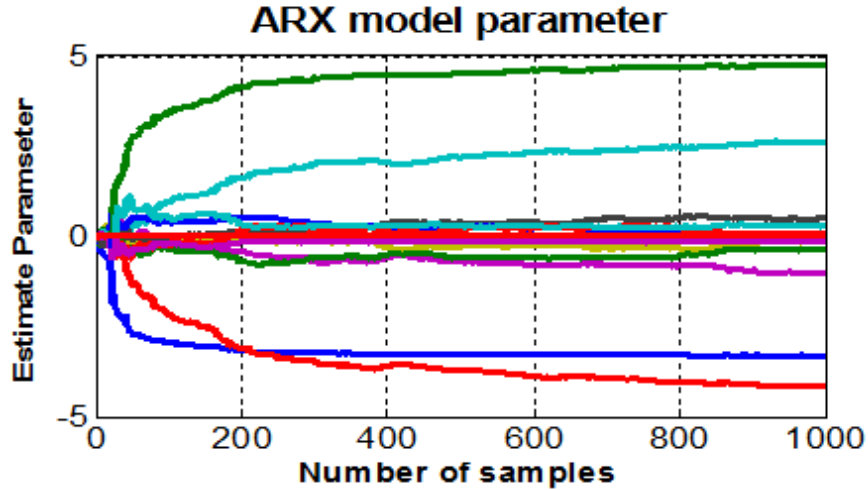

Fig.7. Convergence profile of flexible beam for $12^{\text {th }}$ order

Fig. 8 depicts the comparison between measured output and model predicted output as a validation of the model and Fig. 9 is the same figure which has been zoomed in to show the differences of measured output and predicted output. The measured and predicted output is in good agreement for the model validation test with the mean square error (MSE) of $6.78 \times 10^{-5}$. The identified model is able to simulate the dynamic behavior of the flexible beam as the model close to the actual system.

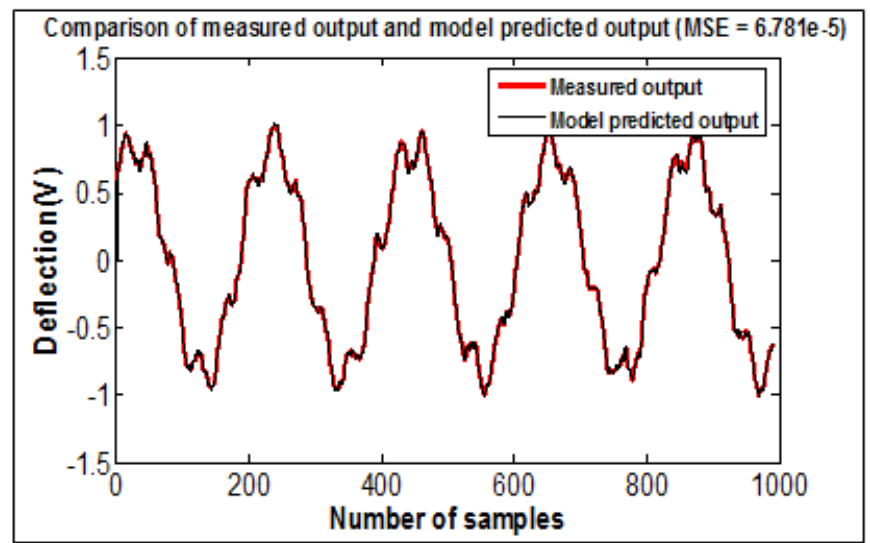

Fig.8. Model validation of measured output and model predicted output

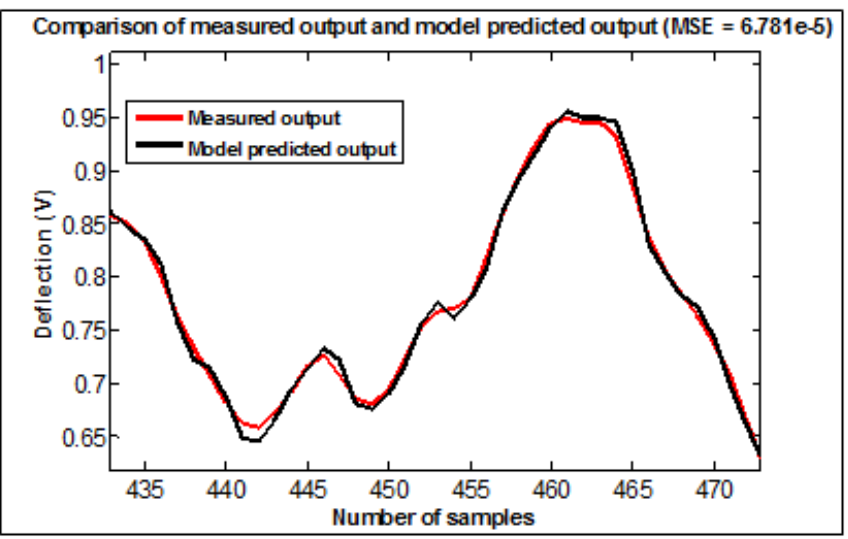

Fig.9. Closed up view of differences between measured and predicted output

From parameter estimation, the parameters of the model structure are substitute into the model structure as the final mathematical model of the flexible beam in obtaining the model predicted output of flexible beam.

\section{V.CONCLUSION}

The model of flexible beam has been developed based on system identification technique using recursive least square algorithm. Before identification process, the resonance test is established to find the natural frequencies of beam and followed by input-output data extraction using square wave signal. The model identification has been estimated from $4^{\text {th }}$ to $12^{\text {th }}$ model order. Based on the result, it can be conclude that the model with highest order shown the lowest value of mean square error. The validation of model via comparison plot between model and measured output revealed that the error is small, which is around $6.78 \times 10^{-5}$.

\section{ACKNOWLEDGEMENTS}

The authors would like to express their gratitude to University Malaysia Perlis for providing facilities to conduct this research. This research was funded by the Ministry of Education Malaysia through Fundamental Research Grant Scheme No. FRGS 9003-00451. 


\section{REFERENCES}

[1]M. S. Hadi, M. H. Hashim and I. Z. M. Darus, "Genetic Modeling of a Rectangular Flexible Plate System with Free-Free-Clamped-Clamped Edges," in IEEE Conference on Control, Systems and Industrial Informatics,(2012), pp. 173-179. https://doi.org/10.1109/CCSII.2012.6470496

[2]Z. M. Darus, A. A. M. Al-Khafaji and M. F. Jamid, "Neuro Modelling of Flexible Plate Structure Rig for Development of Active Vibration Control Algorithm," in International Conference on Mathematical Analytical Modelling and Computer Simulation, (2011).

[3]S. S. Afshari, H. Nobahari, S. Ali and H. Kordkheili, "Experimental Parametric Identification of a Flexible Beam Using Piezoelectric Sensors and Actuators," Journal of Shock and Vibration, (2014).

[4]J. B. L. Anderson, U. Johnsson, K.H Johanson, "A Manual for System Identification," (2000).

[5]M. S. Hadi, I. Z. M. Darus and H. Yatim, "Modeling flexible Plate Structure System with Free-Free-Clamped-Clamped (FFCC) Edges Using Particle Swarm Optimization," in IEEE Symposium on Computers and Informatics, (2013), pp. 39-44. https://doi.org/10.1109/isci.2013.6612372

[6]M. S. Saad, H. Jamaluddin and I. Z. M. Darus, "Active Vibration Control of a Flexible Beam Using System Identification and Controller Tuning by Evolutionary Algorithm," Journal of Vibration and Control, (2013)

[7]H. Nobahari, S.A.H Kordkheili and S. S. Afshari, "Hardware-In-The-Loop Optimization of an Active Vibration Controller in a Flexible Beam Structure Using Evolutionary Algorithms," Journal of Intelligent Materials System and Structures, vol. 25, (2013), pp. 1211-1223 https://doi.org/10.1177/1045389X13502874.

[8]I. Z. M. Darus and M. O. Tokhi, "Parametric and Non-Parametric Identification of a Two Dimensional Flexible Structure," Journal of Low Frequency Noise, Vibration and Active Control, vol. 25, (2006), pp. $119-143$ https://doi.org/10.1260/026309206778494274.

[9]A. R. Tavakolpour, I. Z. Mat Darus, M.O. Tokhi and M. Musa, "Genetic Algorithm-Based Identification of Transfer Function Parameters for a Rectangular Flexible Plate System," Engineering Applications of Artificial Intelligence, vol. 23, (2010), pp. 1388-1397 https://doi.org/10.1016/j.engappai.2010.01.005.

[10] M. Z. Zakaria, M. S. Saad, H. Jamaluddin and R. Ahmad, "Dynamic System Modeling of Flexible Beam System using Multi- Objective Optimization Differential Evolution Algorithm," (2014), pp. 1-4.

[11] A. A. Hussain, M. Al-Khafaji and I. Z. M. Darus, "Evolutionary Algorithm for Identification of a Flexible Single-Link System," Manufacturing Engineering, Automatic Control and Robotics Evolutionary, (2013).

[12] M. N. Azuwir, M. Z. A. Muin and A. H. Adom, "Modelling and Validation of Automotive Engine Fuelled with Palm Oil Biodiesel," International Journal of Engineering and Technology, vol. 3, (2011), pp. $582-586$ https://doi.org/10.7763/IJET.2011.V3.289.

[13] T. N. A. T. Kamaruddin and I. Z. Mat Darus, "System Identification for Internal Combustion Engine Model," in Asia Modelling Symposium, (2012), pp. 17-22. https://doi.org/10.1109/ams.2012.13

[14] M. Yu, N. Feng and E. J. Hahn, "Experimental Evaluation of a Modal Parameter Based System Identification Procedure," Mechanical System and Signal Processing, vol. 68-69, (2016), pp. 302-315 https://doi.org/10.1016/j.ymssp.2015.06.024.

[15] L. R. S. Theisen, H. H. Niemann, I. F. Santos, R. Galeazzi and M. Blanke, "Modelling and identification for control of gas bearings," Mechanical System and Signal Processing, vol. 70-71, (2016), pp. $1150-1170$ https://doi.org/10.1016/j.ymssp.2015.09.016.

[16] A. R. Tavakolpour-Saleh, S. A. R. Nasib, A. Sepasyan and S. M. Hashemi, "Parametric and Nonparametric System Identification of an Experimental Turbojet Engine," Aerospace Science and Technology, vol. 43, (2015), pp. 21-29 https://doi.org/10.1016/j.ast.2015.02.013
About Author (s):

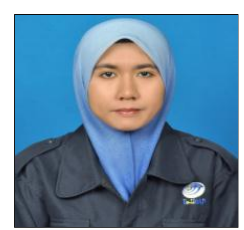

Noor Fadhilah Mat Ros was born in Malaysia in 1990. She received her Diploma and Bachelor Degree of Manufacturing Engineering from University Malaysia Perlis, Malaysia in 2011 and 2014 respectively. She is currently pursuing her Master's degree in University Malaysia Perlis under School of Manufacturing Engineering.

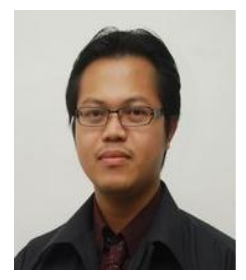

Mohd Sazli Saad was born in Malaysia in 1976. Currently, he is senior lecturer in School of Manufacturing Engineering at University Malaysia Perlis. He graduated from Universiti Teknologi Mara, Malaysia in Bachelor Degree of Electrical Engineering in 2002 and completed his Master Degree (Mechatronic and Automatic Control) Electrical Engineering from Universiti Teknologi Malaysia, in year 2007. In 2014 he received his Phd from University Teknologi Malaysia. 